\title{
Collision Effects in Hydromagneto-Ionic Theory
}

\author{
Hari K. Sen
}

Air Force Cambridge Research Laboratories, Bedford, Mass.

and

\author{
Arne A. Wyller ${ }^{1}$ \\ Williamson Development Company, Inc., West Concord, Mass.
}

(Received May 25, 1964; revised June 8, 1964)

\begin{abstract}
A hydromagneto-ionic theory has been developed within the framework of the Burgers formalism [1958a] which is a microscopic theory based on moments of the Boltzmann transport equation. The effects on electromagnetic wave propagation of electron-electron and electron-proton collisions have been considered to the order of Chapman and Cowling's second approximation. The present theory is an extension of the magneto-ionic theory derived earlier by one of the authors [W yller, 1961] for a fully ionized hydrogen plasma, in that it includes the effects of ion motions. The theory is therefore applicable to the high as well as the low frequency modes of wave propagation, such as the whistler, Alfvén, and the retarded magneto-acoustic modes. Expressions have been derived for the refractive index, absorptivity, the wave polarization, and the zeros and infinities of the refractive index. Numerical applications have been given for the four characteristic modes of low frequency wave propagation, viz, the whistler mode, lower hybrid frequency, ion gyroresonance, and the hydromagnetic mode. Applications of the theory to the solar corona, and future extensions to the terrestrial ionosphere have been indicated.
\end{abstract}

\section{Introduction}

The present paper considers the effect of the ion motions on electromagnetic wave propagation in a uniform plasma with a superposed homogeneous magnetic field. The conventional magneto-ionic theory considers the motions of the electrons only, which is a valid approximation for the high wave frequencies used in ionospheric propagation or for a weakly ionized gas. When the wave frequency goes below the electron gyrofrequency, the extraordinary electromagnetic wave becomes the oblique Alfvén (whistler) mode, and for a further decrease below the ion gyrofrequency the ordinary electromagnetic wave becomes the retarded magneto-acoustic mode. Denisse and Delcroix have given an excellent illustration of this in their book [1961a]. We note that if we take account of the pressure tensor, additional modes will appear. We have not considered these modes in our paper.

Our work differs from most treatments [Schlüter, 1950, 1951; Dungey, 1951; Aström, 1951; Hines, 1953; Piddington, 1956; Gershman, Ginzburg, and

1 Presently at the Institute of Theoretical Astrophysics, University of Oslo, Norway.

$744-758-65 \longrightarrow 7$
Denisov, 1957; Lehnert, 1959; Fejer, 1960; Denisse and Delcroix, 1961b; Allis and Delcroix, 1963] in that it takes account of collisional damping by a microscopic kinetic theory with a proper averaging over the velocity distribution function, of like particle interactions (proton-proton and electronelectron collisions), and of the heat-flow equations.

We have seen that a microscopic treatment through the Boltzmann equation led to significant departures in the propagation factors for electromagnetic waves in the terrestrial ionosphere [Sen and Wyller, 1960; Leinbach, 1962]. Later on, one of us [Wyller, 1961] applied the Burgers formalism [1958a] to find the electrical conductivity for a completely ionized hydrogen plasma. The Burgers formalism proceeds via moments of the Boltzmann transport equation and the associated collision integral. It includes the effects of like particle interactions and of the electron and ion heat flows, and is equivalent to the Chapman and Cowling second approximation [1958]. The present work is an extension of Wyller's [1961] work to include the effects of ion motions.

In a recent work Kantor [1963] has considered the effects of ion motions on magneto-ionic theory without collisions and considered the properties of different directions of wave propagation with respect to the magnetic field. Our work checks with Kantor's formulae for the case of no collisions. 


\section{Basic Equations ${ }^{2}$}

It is well known that the presence of a magnetic field in a plasma introduces an anisotropy in the propagation of electromagnetic waves through the medium. This anisotropy is characterized by the tensorial representation of the associated physical quantities governing the modes of propagation; namely, the electric conductivity and dielectric constant of the medium. After introducing the dielectric tensor (which is easily derived from the conductivity tensor) into the Maxwell equations, one can derive expressions for the refraction, absorption, and polarization of the propagating waves. With this end in view we shall set up in this section the fundamental equations that will enable us to compute the a-c conductivity tensor of the magneto-ionic medium which may transmit electromagnetic waves associated with the oscillations of the ions as well as electrons. In doing so we shall adopt, as we have already said, Burgers' formalism [1958a].

Choosing a rectangular coordinate system $(o, x$, $y, z)$ such that the $z$-axis lies along the uniform applied magnetic field $\vec{H}_{0}$, the electric conductivity tensor can be put into a very simple form. The electric field due to the propagating wave is represented by an a-c field in that medium, $\vec{E} \cos \omega t=$ $\left(E_{x} \vec{i}+E_{y} \vec{j}+E_{z} \vec{k}\right) \cos \omega \mathrm{t}$, where $\vec{i}, \vec{j}$, and $\vec{k}$ are unit vectors along the $x, y$, and $z$ axes and $\omega$ is the excitation frequency of the wave, which is assumed to remain constant during the propagation of the wave inside the medium.

We shall assume the plasma medium to be homogeneous with no pressure and density gradients. We shall consider a fully ionized hydrogen plasma containing electrons and protons in equal number densities, $n_{1}=n_{2}$, throughout the medium. The subscripts 1 and 2 will be used consistently to denote protons and electrons respectively.

The assumptions made above bring about a considerable simplification in the Burgers formalism. There is a simple relation between Burgers" "friction coefficient" $K_{12}=K_{21}=K$ and the mean electronproton collision frequency $\bar{\nu}$, namely,

$$
K=n_{2} m_{2} \bar{\nu} \text {, where } \bar{\nu}=\frac{\int_{0}^{\infty} \nu e^{-m_{2} v_{2}^{2} / 2 k T} v_{2}^{4} d v_{2}}{\int_{0}^{\infty} e^{-m_{2} v_{2}^{2} / 2 k T} v_{2}^{4} d v_{2}} \text {, }
$$

or

$$
\bar{\nu}=\frac{2}{3 \sqrt{\pi}} \frac{\omega_{0}^{2} e_{2}^{2}}{k T}\left(\frac{m_{2}}{2 k T}\right)^{1 / 2} \ln \cdot \Lambda, \Lambda=\frac{3 k T}{e_{2}^{2}} r_{D}
$$

Here $r_{D}$ is the Debye length, and $\omega_{0}$ is the electron plasma frequency.

In order to assess the full implications of the momentum and heat transfer equations of the elec-

2 Sections 2,3 and 4 were performed by one of us (A.A.W.) while on summer, leave in 1963 from the Institute of Theoretical Astrophysics, University of OsloNorway, to Williamson Development Company, Inc., West Concord, Mass. trons and protons, we state here some of the physical parameters of Burgers' theory that are relevant to the conditions of our problem. For a binary gas with known relative velocities of the colliding particles " $g$ " the collision cross section is given by:

$$
S_{s t}^{(l)}=S_{t s}^{(l)}=2 \pi \int_{0}^{r_{D}}\left(1-\cos ^{l} \chi\right) b d b .
$$

where $b$ and $\chi$ are Chapman and Cowling's [1958] collision parameters. In particular, $l=1$ for momentum transfer with which we are primarily concerned here. The average collision cross section for all possible relative velocities is

$$
Z_{s t}^{(l j)}=Z_{t s}^{(l j)}=\frac{4}{\sqrt{\pi}}(\mu / 2 k T)^{j+2} \int_{0}^{\infty} g^{2 j+3} e^{-\mu g^{2} / 2 k T} S_{s t}^{(l)} d g .
$$

Here the subscripts " $s$ " and " $t$ " refer to the species of the colliding particles and $\mu$ is the reduced mass. The superscript " $l$ " refers to the angular dependence while " $j "$ refers to the velocity dependence of the collision cross section. In terms of these average collision cross sections, the quantity defined by

$$
z_{s t}=1-\frac{2}{5} \frac{Z_{s t}^{(12)}}{Z_{s t}^{(11)}}
$$

reduces to the value $3 / 5$ for Coulomb interactions.

In order to be able to estimate the effects of collisions between like and unlike particles separately Burgers [1958b] introduces the following collision factors which turn out to be proportional to the average collision frequencies,

$$
\kappa=\frac{4}{5} \frac{m_{1} m_{2}}{\left(m_{1}+m_{2}\right)^{2}} \frac{Z_{12}^{(22)}}{Z_{12}^{(11)}} \quad \text { (electron-proton collisions) }
$$

$\kappa_{1}=\frac{2}{5}\left(\frac{m_{1}+m_{2}}{2 m_{2}}\right)^{1 / 2} \frac{Z_{11}^{(22)}}{Z_{12}^{(11)}}$ (proton-proton collision.)

$\kappa_{2}=\frac{2}{5}\left(\frac{m_{1}+m_{2}}{2 m_{1}}\right)^{1 / 2} \frac{Z_{22}^{(22)}}{Z_{12}^{(11)}} \quad$ (electron-electron collisions).

It will be seen later that the auxiliary quantity $\xi$ which incorporates the effects of electron-proton and electron-electron collisions enters into the electrical conductivity tensor. It is defined by

$$
\xi=\kappa+\kappa_{2}+\gamma Y_{2} ; \gamma=\frac{m_{2}}{m_{1}}
$$

and $Y_{2}$ is a function of $\kappa$ only.

Though the proton-proton collisions do enter into the equations of the heat flow through the factor $\kappa_{1}$, their net effects in the electrical conductivity tensor vanish [Spitzer, 1956; Marshall, 1957]. On the other hand, in thermal conductivity the dissipative effects due to ion-ion encounters is not negligible [Rosenbluth and Kaufman, 1958; Wyller, 1963a].

Burgers' momentum and heat flow equations in their time-dependent forms will be our fundamental 
set of equations for the electrons and protons. We will rewrite Burgers' equations [1958c] as follows.

Momentum equations for electrons:

$$
\begin{aligned}
i \omega u_{2 x}-\Gamma_{2 x}+s_{2} u_{2 y} & =\bar{\nu}\left(u_{1 x}-u_{2 x}\right)+z \bar{\nu} r_{2 x}-z \gamma \bar{\nu} r_{1 x}, \\
i \omega u_{2 y}-\Gamma_{2 y}-s_{2} u_{2 x} & =\bar{\nu}\left(u_{1 y}-u_{2 y}\right)+z \bar{\nu} r_{2 y}-z \gamma \bar{\nu} r_{1 y}, \\
i \omega u_{2 z}-\Gamma_{2 z} & =\bar{\nu}\left(u_{1 z}-u_{2 z}\right)+z \bar{\nu} r_{2 z}-z \gamma \bar{\nu} r_{1 z} .
\end{aligned}
$$

Momentum equations for protons:

$$
\begin{aligned}
i \omega u_{1 x}-\Gamma_{1 x}-s_{1} u_{1 y} & =-\gamma \bar{\nu}\left(u_{1 x}-u_{2 x}\right)-z \gamma \bar{\nu} r_{2 x}+z \gamma^{2} \bar{\nu} r_{1 x}, \\
i \omega u_{1 y}-\Gamma_{1 y}+s_{1} u_{1 x} & =-\gamma \bar{\nu}\left(u_{1 y}-u_{2 y}\right)-z \gamma \bar{\nu} r_{2 y}+z \gamma^{2} \bar{\nu} r_{1 y}, \\
i \omega u_{1 z}-\Gamma_{1 z} & =-\gamma \bar{\nu}\left(u_{1 z}-u_{2 z}\right)-z \gamma \bar{\nu} r_{2 z}+z \gamma^{2} \bar{\nu} r_{1 z} .
\end{aligned}
$$

Heat flow equations for electrons:

$$
\begin{aligned}
i \omega r_{2 x}+s_{2} r_{2 y} & =-\frac{5}{2} z \bar{\nu}\left(u_{1 x}-u_{2 x}\right)-\xi \bar{\nu} r_{2 x}+\frac{27}{10} \gamma \bar{\nu} r_{1 x}, \\
i \omega r_{2 y}-s_{2} r_{2 x} & =-\frac{5}{2} z \bar{\nu}\left(u_{1 y}-u_{2 y}\right)-\xi \bar{\nu} r_{2 y}+\frac{27}{10} \gamma \bar{\nu} r_{1 y}, \\
i \omega r_{2 z} & =-\frac{5}{2} z \bar{\nu}\left(u_{1 z}-u_{2 z}\right)-\xi \bar{\nu} r_{2 z}+\frac{27}{10} \gamma \bar{\nu} r_{1 z} .
\end{aligned}
$$

Heat flow equations for protons:

$$
\begin{aligned}
i \omega r_{1 x}-s_{1} r_{1 y} & =\frac{5}{2} z \gamma^{2} \bar{\nu}\left(u_{1 x}-u_{2 x}\right)-\gamma \kappa_{1} \bar{\nu} r_{1 x}+\frac{27}{10} \gamma^{2} \bar{\nu} r_{2 x}, \\
i \omega r_{1 y}+s_{1} r_{1 x} & =\frac{5}{2} z \gamma^{2} \bar{\nu}\left(u_{1 y}-u_{2 y}\right)-\gamma \kappa_{1} \bar{\nu} r_{1 y}+\frac{27}{10} \gamma^{2} \bar{\nu} r_{2 y}, \\
i \omega r_{1 z} & =\frac{5}{2} z \gamma^{2} \bar{\nu}\left(u_{1 z}-u_{2 z}\right)-\gamma \kappa_{1} \bar{\nu} r_{1 z}+\frac{27}{10} \gamma^{2} \bar{\nu} r_{2 z} .
\end{aligned}
$$

In the preceding equations the quantity $i \omega$ appears because of the time dependence of the particle velocity and heat flow velocity respectively induced by the alternating electric field $\overrightarrow{\mathscr{E}}=\overrightarrow{\mathscr{E}}_{0} e^{i \omega t}$. The mean flow velocity component, $u_{s h}$, is the mean value of the particle velocities, $\xi_{s h}$,

$$
u_{s h}=\bar{\xi}_{s h} .
$$

Also the accelerations due to the electric field are denoted by

$$
\vec{\Gamma}_{i}=\frac{e_{i}}{m_{i}} \overrightarrow{\mathscr{E}} ; \quad e_{1}=+e ; \quad e_{2}=-e
$$

so that

$$
\vec{\Gamma}_{1}=-\gamma \vec{\Gamma}_{2}, \gamma=\frac{m_{2}}{m_{1}}
$$

The gyrofrequencies are defined by

$$
s_{i}=\left|\frac{e_{i}}{m_{i}} H_{z}\right| .
$$

The foregoing set of 12 equations in 12 unknowns will now be solved to obtain the current flow components $\left(u_{1 h}-u_{2 h}\right)$. Then the conductivity tensor will be found from the usual formula:

$$
\vec{I}=-n_{2} e_{2} \vec{w}=\sigma \cdot \vec{E}
$$

\section{Solution for Electrical Conductivity Along the Magnetic Field}

The momentum and heat flow equations for both electrons and protons along the $z$ axis are:

$$
\begin{gathered}
i \omega u_{2 z}-\Gamma_{2 z}=\bar{\nu}\left(u_{1 z}-u_{2 z}\right)+z \bar{\nu} r_{2 z}-z \gamma \bar{\nu} r_{1 z}, \\
i \omega u_{1 z}-\Gamma_{1 z}=-\gamma \bar{\nu}\left(u_{1 z}-u_{2 z}\right)-z \gamma \bar{\nu} r_{2 z}+z \gamma^{2} \bar{\nu} r_{1 z}, \\
i \omega r_{2 z}=-\frac{5}{2} z \bar{\nu}\left(u_{1 z}-u_{2 z}\right)-\xi \bar{\nu} r_{2 z}+\frac{27}{10} \gamma \bar{\nu} r_{1 z}, \\
i \omega r_{1 z}=\frac{5}{2} z \gamma^{2} \bar{\nu}\left(u_{1 z}-u_{2 z}\right)-\gamma \kappa_{1} \bar{\nu} r_{2 z}+\frac{27}{10} \gamma^{2} \bar{\nu} r_{2 z} .
\end{gathered}
$$

According to Burgers we neglect the terms $z \gamma \bar{\nu} r_{1 z}$, $z \gamma^{2} \bar{\nu} r_{1 z}$, and $\frac{27}{10} \gamma \bar{\nu} r_{1 z}$ for $\gamma r_{1 z}<<r_{2 i}$. This can be verified by solving for $r_{1 h}$ and $r_{2 h}$ from the basic equations of the last section.

From (16a) we have

$$
r_{2 z}=-\frac{5}{2} z \bar{\nu} \frac{\left(u_{1 z}-u_{2 z}\right)}{(\xi \bar{\nu}+i \omega)} .
$$

Upon inserting (17) into (15a, b) we find that

$$
\begin{gathered}
i \omega u_{2 z}-\Gamma_{2 z}=\bar{\nu}\left(u_{1 z}-u_{2 z}\right)-\frac{5}{2} \frac{z^{2} \bar{\nu}^{2}\left(u_{1 z}-u_{2 z}\right)}{(\xi \bar{\nu}+i \omega)} \\
i \omega u_{1 z}-\Gamma_{1 z}=-\gamma \bar{\nu}\left(u_{1 z}-u_{2 z}\right)+\frac{5}{2} \frac{\gamma z^{2} \bar{\nu}^{2}\left(u_{1 z}-u_{2 z}\right)}{(\xi \bar{\nu}+i \omega)} .
\end{gathered}
$$

Defining the current flow velocity as $\vec{w}=\overrightarrow{u_{1}}-\vec{u}_{2}$ (14) we have

$$
w_{z}=u_{1 z}-u_{2 z} .
$$

Thus we get from $(18 \mathrm{a}, \mathrm{b})$,

$$
i \omega w_{z}+(1+\gamma) \Gamma_{2 z}=-(1+\gamma) \bar{\nu} w_{z}+(1+\gamma) \frac{5}{2} \frac{z^{2} \bar{\nu}^{2} w_{2}}{(\xi \bar{\nu}+i \omega)} .
$$

Now solving for $w_{z}$ with separation of real and 
imaginary parts we obtain

$$
\begin{aligned}
& w_{z^{\prime}}^{\prime}\left[(1+\gamma) \bar{\nu}\left(1-\frac{5}{2} \frac{z^{2} \bar{\nu}^{2} \xi}{\xi^{2} \bar{\nu}^{2}+\omega^{2}}\right)\right. \\
& \left.\quad+i \omega\left(1+(1+\gamma) \frac{5}{2} \frac{z^{2} \bar{\nu}^{2}}{\xi^{2} \bar{\nu}^{2}+\omega^{2}}\right)\right]=-(1+\gamma) \Gamma_{2 z} .
\end{aligned}
$$

At this stage we introduce simplifying notations to condense the final expression for the conductivity tensor component along the z-direction and to elucidate therein the effects of electron-electron and electron-proton collisions; we set

$$
\delta_{3}=\frac{5}{2} \frac{z^{2}}{\xi} \frac{\xi^{2} \bar{\nu}^{2}}{\xi^{2} \bar{\nu}^{2}+\omega^{2}} \text { and } \bar{\nu}_{3}=\frac{\left(1-\delta_{3}\right) \bar{\nu}}{\left[1+(1+\gamma) \frac{\delta_{3}}{\xi}\right]} \text {. }
$$

The component of the current vector in the $z$-direction is then given by

$$
\begin{array}{r}
I_{z}=-n_{2} e_{2} w_{z}=\frac{(1+\gamma) \sigma_{0} \mathscr{E}_{z}}{\left[1+(1+\gamma) \frac{\delta_{3}}{\xi}\right]\left[(1+\gamma) \nu_{3}+i \omega\right]} \\
\sigma_{0}=\frac{\omega_{0}^{2}}{4 \pi}
\end{array}
$$

and the conductivity tensor component in the $z$-direction is finally obtained

$$
\sigma_{z z}=\frac{(1+\gamma) \sigma_{0}}{\left[1+(1+\gamma) \frac{\delta_{3}}{\xi}\right]\left[(1+\gamma) \nu_{3}+i \omega\right]} .
$$

When we compare this tensor component with the corresponding Lorentz tensor component $\sigma_{0}$ / $(\bar{\nu}+i \omega)$, we find that the ion-motions affect the conductivity along the z-direction through the factor $(1+\gamma)$ while the electron-proton and electron-electron collisions through $\delta_{3}$ and $\nu_{3}$. In particular, the expression (24) checks with Spitzer's d-c conductivity [1962], when $\gamma$ and $\omega$ are set equal to zero.

\section{Solutions for Conductivities Perpendicular to the Magnetic Field}

The magnetic field couples the $x$ and $y$ components of the momentum and heat flow equations, making the solution for the flow components $u_{i x}$ and $u_{i y}$ somewhat awkward to obtain. We may avoid this difficulty by introducing a coordinate system defined by the unit bi-vectors [Menzel, 1961]:

$$
\vec{\alpha}=\frac{1}{\sqrt{2}}(\vec{i}+i \vec{j}), \vec{\beta}=\frac{1}{\sqrt{2}}(\vec{i}-i \vec{j}), \vec{\gamma}=\vec{k} .
$$

Now we may express the electric field as, omitting the time dependence for simplicity,

$$
\begin{aligned}
& \overrightarrow{\mathscr{E}}=\mathscr{E}_{x} \vec{i}+\mathscr{E}_{y} \vec{j}+\mathscr{E}_{z} \vec{k}=\frac{1}{\sqrt{2}}\left(\mathscr{E}_{x}-i \mathscr{E}_{y}\right) \vec{\alpha}+\frac{1}{\sqrt{2}} \\
& \left(\mathscr{E}_{x}+i \mathscr{E}_{y}\right) \vec{\beta}+\mathscr{E}_{z} \vec{\gamma}, \\
& =\mathscr{E}_{l} \vec{\alpha}+\mathscr{E}_{r} \vec{\beta}+\mathscr{E}_{z} \vec{\gamma}
\end{aligned}
$$

Allis used a similar coordinate system in his formulation of the conductivity tensor for plasmas [Allis, 1956]. $\mathscr{E}_{l}$ corresponds to the left rotating field component and $\mathscr{E}_{r}$ to the right rotating component.

We also write the flow components in this coordinate system

$$
\begin{aligned}
& u_{i l}=\frac{1}{\sqrt{2}}\left(u_{i x}-i u_{i y}\right), u_{i r}=\frac{1}{\sqrt{2}}\left(u_{i x}+i u_{i y}\right) \\
& r_{i l}=\frac{1}{\sqrt{2}}\left(r_{i x}-i r_{i y}\right), r_{i r}=\frac{1}{\sqrt{2}}\left(r_{i x}+i r_{i y}\right) .
\end{aligned}
$$

We begin by solving for $w_{r}$ from the rewritten momentum and heat flow equations

$$
\begin{gathered}
i \omega u_{2 r}-\Gamma_{2 r}-i s_{2} u_{2 r}=\bar{\nu}\left(u_{1 r}-u_{2 r}\right)+z \bar{\nu} r_{2 r} \\
i \omega u_{1 r}-\Gamma_{1 r}+i s_{1} u_{1 r}=-\gamma \bar{\nu}\left(u_{1 r}-u_{2 r}\right)-z \gamma \bar{\nu} r_{2 r} \\
i \omega r_{2 r}-i s_{2} r_{2 r}=-\frac{5}{2} z \bar{\nu}\left(u_{1 r}-u_{2 r}\right)-\xi \bar{\nu} r_{2 r} \\
i \omega r_{1 r}+i s_{1} r_{1 r}=\frac{5}{2} z \gamma^{2} \bar{\nu}\left(u_{1 r}-u_{2 r}\right)-\gamma \kappa_{1} \bar{\nu} r_{1 r}+\frac{27}{10} \gamma^{2} \bar{\nu} r_{2 r} .
\end{gathered}
$$

From (28a) we have

$$
\bar{\nu}\left(u_{1 r}-u_{2 r}\right)=i \omega u_{2 r}-\Gamma_{2 r}-i s_{2} u_{2 r}-z \bar{\nu} r_{2 r},
$$

which yields $r_{2 r}$ when put into (29a)

$$
r_{2 r}=\frac{5}{2} z\left[\frac{i\left(s_{2}-\omega\right) u_{2 r}+\Gamma_{2 r}}{\bar{\nu}\left(\xi-\frac{5}{2} z^{2}\right)-i\left(s_{2}-\omega\right)}\right] .
$$

Putting expression (30) for $\nu\left(u_{1 r}-u_{2 r}\right)$ into we obtain

$$
u_{1 r}=\gamma \frac{\left(s_{2}-\omega\right)}{\left(s_{1}+\omega\right)} u_{2 r}
$$

since $\Gamma_{1 r}=-\gamma \Gamma_{2 r}$. The electric field and heat flow terms have dropped out. At this point we may return to (28a) with these expressions for $u_{1 r}$ and $r_{2 r}$ and solve uniquely for

$$
u_{2 r}=\frac{-\left[\xi \bar{\nu}-i\left(s_{2}-\omega\right)\right] \Gamma_{2 r}}{\left\{\left(s_{2}-\omega\right)^{2}+\left[\bar{\nu}\left(\xi-\frac{5}{2} z^{2}\right)-i\left(s_{2}-\omega\right)\right]\left[\gamma \bar{\nu} \frac{\left(s_{2}-\omega\right)}{\left(s_{1}+\omega\right)}-\bar{\nu}\right]+i \xi \bar{\nu}\left(s_{2}-\omega\right)\right\}}
$$


The total current flow associated with the right rotating electric field is then the difference of the electron and proton flows

$$
w_{r}=u_{1 r}-u_{2 r}=\left[\gamma \frac{\left(s_{2}-\omega\right)}{\left(s_{1}+\omega\right)}-1\right] u_{2 r} .
$$

For subsequent algebraic simplification we introduce the symbols

$$
\alpha=1+\gamma \frac{\left(\omega-s_{2}\right)}{\left(\omega+s_{1}\right)} \text { and } A=\left[\bar{\nu}\left(\xi-\frac{5}{2} z^{2}\right)-i\left(s_{2}-\omega\right)\right]
$$

which gives

$$
w_{r}=\frac{\alpha\left[\xi \bar{\nu}-i\left(s_{2}-\omega\right)\right] \Gamma_{2 r}}{\left[\left(s_{2}-\omega\right)^{2}-\alpha \bar{\nu} A+i \xi \bar{\nu}\left(s_{2}-\omega\right)\right]} .
$$

Expression (35) is equivalent to

$$
w_{r}=\frac{(+\alpha) \Gamma_{2 r}}{\frac{\left[\left(s_{2}-\omega\right)^{2}-\alpha \bar{\nu} A+i \xi \bar{\nu}\left(s_{2}-\omega\right)\right]\left[\xi \bar{\nu}+i\left(s_{2}-\omega\right)\right]}{\left[\xi \bar{\nu}+i\left(s_{2}-\omega\right)\right]\left[\xi \bar{\nu}-i\left(s_{2}-\omega\right)\right]}} .
$$

On separating the real and imaginary parts in the denominator, we have

Now we introduce a new symbol $\delta_{1}$ and the expression for $w_{r}$ becomes

$$
\begin{aligned}
& w_{r}=\frac{(-\alpha) \Gamma_{2 r}}{\alpha \bar{\nu}\left(1-\delta_{1}\right)+i\left(\omega-s_{2}\right)\left(1+\frac{\alpha \delta_{1}}{\xi}\right)}, \\
& \delta_{1}=\frac{5}{2} \frac{z^{2}}{\xi} \frac{\xi^{2} \bar{\nu}^{2}}{\xi^{2} \bar{\nu}^{2}+\left(\omega-s_{2}\right)^{2}} .
\end{aligned}
$$

We can repeat the foregoing analysis and obtain the flow for the case of the left rotating electric field

$$
w_{l}=\frac{-\beta \Gamma_{2 l}}{\beta \bar{\nu}\left(1-\delta_{2}\right)+i\left(\omega+s_{2}\right)\left(1+\beta \frac{\delta_{2}}{\xi}\right)},
$$

where, as before, we have simplified the expression with the symbols

$$
\delta_{2}=\frac{5}{2} \frac{z^{2}}{\xi} \frac{\xi^{2} \bar{\nu}^{2}}{\xi^{2} \bar{\nu}^{2}+\left(\omega+s_{2}\right)^{2}} \text { and } \beta=1+\gamma \frac{\left(\omega+s_{2}\right)}{\left(\omega-s_{1}\right)} .
$$

From the foregoing, we obtain the total current

$$
\vec{I}=-n_{2} e_{2}\left(w_{\imath} \vec{\alpha}+w_{r} \vec{\beta}+w_{z} \vec{\gamma}\right)
$$

$$
w_{r}=\frac{(+\alpha) \Gamma_{2 r}}{\left\{-\alpha \bar{\nu}\left[1-\frac{5}{2} \frac{z^{2} \bar{\nu}^{2} \xi}{\xi^{2} \bar{\nu}^{2}+\left(\omega-s_{2}\right)^{2}}\right]-i\left(\omega-s_{2}\right)\left[1+\alpha \frac{5}{2} \frac{z^{2} \bar{\nu}^{2}}{\xi^{2} \bar{\nu}^{2}+\left(\omega-s_{2}\right)^{2}}\right]\right\}} .
$$

Rather than directly solve for $u_{x}$ and $u_{y}$, we derive the electrical conductivity tensor in a form analogous to that of the Lorentz tensor form [Sen and Wyller, 1960]. We first introduce the symbols

$$
\gamma_{l}=\frac{w_{l}}{-\Gamma_{2 l}}, \gamma_{r}=\frac{w_{r}}{-\Gamma_{2 r}}, \gamma_{z}=\frac{w_{z}}{-\Gamma_{2 z}},
$$

which let us rewrite expression (40) as

$\vec{I}=n_{2} e_{2}\left[\frac{1}{\sqrt{2}} \gamma_{l} \Gamma_{2 l}(\vec{i}+\overrightarrow{i j})+\frac{1}{\sqrt{2}} \gamma_{r} \Gamma_{2 r}(\vec{i}-\overrightarrow{i j})+\gamma_{2} \Gamma_{2 z} \vec{k}\right]$

and

$$
\begin{aligned}
\vec{I} & =I_{x} \vec{i}+I_{y} \vec{j}+I_{z} \vec{k}=\frac{\omega_{0}^{2}}{8 \pi}\left[\gamma_{l}\left(\mathscr{E}_{x}-i \mathscr{E}_{y}\right) \vec{i}+\gamma_{l}\left(\mathscr{E}_{y}+i \mathscr{E}_{x}\right) \vec{j}\right. \\
& \left.+\gamma_{r}\left(\mathscr{E}_{x}+i \mathscr{E}_{y}\right) \vec{i}+\gamma_{r}\left(\mathscr{E}_{y}-i \mathscr{E}_{x}\right) \vec{j}+2 \gamma_{z} \mathscr{E}_{z} \vec{k}\right],
\end{aligned}
$$

or, in matrix form

$$
\left\{\begin{array}{l}
I_{x} \\
I_{y} \\
I_{z}
\end{array}\right\}=\frac{\omega_{0}^{2}}{8 \pi}\left\{\begin{array}{ccc}
\left(\gamma_{l}+\gamma_{r}\right) & i\left(\gamma_{r}-\gamma_{l}\right) & 0 \\
-i\left(\gamma_{r}-\gamma_{l}\right) & \left(\gamma_{l}+\gamma_{r}\right) & 0 \\
0 & 0 & 2 \gamma_{z}
\end{array}\right\}\left\{\begin{array}{l}
\mathscr{E}_{x} \\
\mathcal{E}_{y} \\
\mathcal{E}_{z}
\end{array}\right\} .
$$

Then the complete electrical conductivity tensor in the hydromagneto-ionic theory becomes

$$
\stackrel{\rightarrow}{\vec{\sigma}}=\frac{\omega_{0}^{2}}{8 \pi}\left\{\begin{array}{ccc}
\left(\gamma_{l}+\gamma_{r}\right) & i\left(\gamma_{r}-\gamma_{l}\right) & 0 \\
-i\left(\gamma_{r}-\gamma_{l}\right) & \left(\gamma_{l}+\gamma_{r}\right) & 0 \\
0 & 0 & 2 \gamma_{z}
\end{array}\right\}
$$

where

$\gamma_{r}=\frac{\alpha}{\left(1+\alpha \frac{\delta_{1}}{\xi}\right)\left[\alpha \nu_{1}+i\left(\omega-s_{2}\right)\right]}, \quad \alpha=1+\gamma \frac{\left(\omega-s_{2}\right)}{\left(\omega+s_{1}\right)}$

$\gamma_{l}=\frac{\beta}{\left(1+\beta \frac{\delta_{2}}{\xi}\right)\left[\beta \nu_{2}+i\left(\omega+s_{2}\right)\right]}, \quad \beta=1+\gamma \frac{\left(\omega+s_{2}\right)}{\left(\omega-s_{1}\right)}$

$$
\begin{aligned}
& \gamma_{z}=\frac{(1+\gamma)}{\left[1+(1+\gamma) \frac{\delta_{3}}{\xi}\right]\left[(1+\gamma) \nu_{3}+i \omega\right]}, \quad \gamma=\frac{m_{2}}{m_{1}} \\
& \nu_{1}=\frac{\left(1-\delta_{1}\right) \bar{\nu}}{\left(1+\alpha \frac{\delta_{1}}{\xi}\right)} ; \nu_{2}=\frac{\left(1-\delta_{2}\right) \bar{\nu}}{\left(1+\beta \frac{\delta_{2}}{\xi}\right)} ; \nu_{3}=\frac{\left(1-\delta_{3}\right) \bar{\nu}}{\left[1+(1+\gamma) \frac{\delta_{3}}{\xi}\right]} .
\end{aligned}
$$


Thus we have been able to express the electrical conductivity tensor containing ion-motion, electronelectron and electron-proton collisions into a form which is similar to that of the Lorentz tensor [Sen and Wyller, 1960a].

When $\gamma$ goes to zero, (45) reduces to a form of the electrical conductivity tensor [Wyller, 1961] valid for high frequencies (conventional magneto-ionic theory).

The dielectric tensor is derived from the relation

for which we find

$$
\vec{\epsilon}=\overrightarrow{1}+\frac{4 \pi}{i \omega} \vec{\sigma}
$$

$$
\begin{array}{r}
\epsilon_{\mathrm{I}}=\left(1-a_{11}\right)-i b_{11}, \epsilon_{\mathrm{II}}=\frac{1}{2}\left(f_{11}-d_{11}\right)+\frac{i}{2}\left(c_{11}-e_{11}\right), \\
\epsilon_{\mathrm{III}}=\left[a_{11}-\frac{1}{2}\left(c_{11}+e_{11}\right)\right]+i\left[b_{11}-\frac{1}{2}\left(f_{11}+d_{11}\right)\right],
\end{array}
$$

where

$$
\begin{aligned}
& a_{11}=\frac{\omega_{0}^{2}(1+\gamma)}{\left[1+(1+\gamma) \frac{\delta_{3}}{\xi}\right]\left[(1+\gamma)^{2} \nu_{3}^{2}+\omega^{2}\right]}, \\
& b_{11}=\frac{\omega_{0}^{2}(1+\gamma)^{2} \nu_{3}}{\omega\left[1+(1+\gamma) \frac{\left.\delta_{3}\right]}{\xi}\right]\left[(1+\gamma)^{2} \nu_{3}^{2}+\omega^{2}\right]}, \\
& c_{11}=\frac{\omega\left(1+\alpha \frac{\delta_{1}}{\xi}\right)\left[\alpha^{2} \nu_{1}^{2}+\left(\omega-s_{2}\right)^{2}\right]}{d_{11}=}, \\
& e_{11}=\frac{\omega\left(1+\alpha \frac{\delta_{1}}{\xi}\right)\left[\alpha^{2} \alpha_{1}^{2}+\left(\omega-s_{2}\right)^{2}\right]}{\omega\left(1+\beta \frac{\delta_{2}}{\xi}\right)\left[\beta^{2} \nu_{2}^{2}+\left(\omega+s_{2}\right)^{2}\right]}, \\
& f_{11}=\frac{\omega_{0}^{2} \beta^{2} \nu_{2}}{\omega\left(1+\beta \frac{\delta_{2}}{\xi}\right)\left[\beta^{2} \nu_{2}^{2}+\left(\omega+s_{2}\right)^{2}\right]} .
\end{aligned}
$$

Retaining the Sen and Wyller formalism [1960] we find that the formulae for the complex refractive index $c / u$ and the polarization $R$ are unaltered in form except that the constants $A, B, C$, etc., are now functions of $\epsilon_{\mathrm{I}}, \epsilon_{\mathrm{II}}$, and $\epsilon_{\mathrm{III}}$ defined in (48). This leads us to a new formulation of the hydromagneto-ionic theory which permits the study of low frequency modes such as the Alfvén mode and the retarded magneto-acoustical mode with electronelectron and electron-proton collisions included. For convenience we recapitulate [Sen and Wyller, 1960b] $\frac{c^{2}}{u^{2}}=\left(n-i \frac{c \kappa}{\omega}\right)^{2}=\frac{A+B \sin ^{2} \phi \pm\left(B^{2} \sin ^{4} \phi-C^{2} \cos ^{2} \phi\right)^{1 / 2}}{D+E \sin ^{2} \phi}$,

$A=2 \epsilon_{\mathrm{I}}\left(\epsilon_{\mathrm{I}}+\epsilon_{\mathrm{III}}\right), B=\epsilon_{\mathrm{III}}\left(\epsilon_{\mathrm{I}}+\epsilon_{\mathrm{III}}\right)+\epsilon_{\mathrm{II}}^{2}, C=2 \epsilon_{\mathrm{I}} \epsilon_{\mathrm{II}}$,

$$
D=2 \epsilon_{\mathrm{I}}, E=2 \epsilon_{\mathrm{III}},
$$

$R=-\frac{\mathscr{E} y}{\mathscr{E} z}=-\frac{B \sin ^{2} \phi \mp\left(B^{2} \sin ^{4} \phi-C^{2} \cos ^{2} \phi\right)^{1 / 2}}{C \cos \phi}$.

Here $n$ is the real refractive index, $c_{\kappa} / \omega$ is the absorptivity, and $\phi$ is the angle between the direction of the magnetic field and the direction of propagation.

\section{Zeroes and Infinities of the Refractive Index}

In the formula (49) the complex refractive index $\bar{n}=n-i \frac{c \kappa}{\omega}$ satisfies a biquadratic equation

$\bar{n}^{4}+\bar{n}^{2} \frac{\left(A+B \sin ^{2} \phi\right)}{\left(D+E \sin ^{2} \phi\right)}+\frac{A^{2}+2 A B \sin ^{2} \phi+C^{2} \cos ^{2} \phi}{\left(D+E \sin ^{2} \phi\right)^{2}}=0$.

Hence the condition that $\bar{n}$ may have a zero root is that

$$
A^{2}+2 A B \sin ^{2} \phi+C^{2} \cos ^{2} \phi=0
$$

provided that $D+E \sin ^{2} \phi \neq 0$. After some algebraic simplifications the above two expressions reduce respectively to

$$
\begin{gathered}
\epsilon_{\mathrm{I}}\left[\left(\epsilon_{\mathrm{I}}+\epsilon_{\mathrm{III}}\right)^{2}+\epsilon_{\mathrm{II}}^{2}\right]\left(\epsilon_{\mathrm{I}}+\epsilon_{\mathrm{III}} \sin ^{2} \phi\right)=0 \\
\epsilon_{\mathrm{I}}+\epsilon_{\mathrm{III}} \sin ^{2} \phi \neq 0 .
\end{gathered}
$$

By virtue of the restriction (53), all the zero roots of $\bar{n}$ will be given by the following three equations,

$$
\text { (a) } \epsilon_{\mathrm{I}}=0 \text {, (b) } \epsilon_{\mathrm{I}}+\epsilon_{\mathrm{III}}-i \epsilon_{\mathrm{II}}=0 \text {, (c) } \epsilon_{\mathrm{I}}+\epsilon_{\mathrm{III}}+i \epsilon_{\mathrm{II}}=0 \text {. }
$$

These equations are independent of the propagation angle " $\phi "$. Assigning the appropriate values to $\epsilon_{\mathrm{I}}$ from (48) we find that (a) will hold when $a_{11}=1$ and $b_{11}=0$ simultaneously. The latter condition means that either $\bar{\nu}=0$, i.e., there is no collision, or else that $\delta_{3}=1$, which gives an imaginary value to the collision parameter $Z=\bar{\nu} / \omega$ and is therefore inadmissible. The first condition with $\bar{\nu}=0$ yields a cutoff for $n$ at

$$
X=\frac{1}{1+\gamma} .
$$

Similar treatment to (b) and (c) yields cutoff values of $n$ without collisions at

$$
X=\frac{(1+Y)(1-\gamma Y)}{1+\gamma},
$$


and

respectively.

$$
X=\frac{(1-Y)(1+\gamma Y)}{1+\gamma}
$$

In (54) and (55), $X=\omega_{0}^{2} / \omega^{2}$ and $Y=s_{2} / \omega$. The cutoff values are not attained when collisions are present. These cutoff values will be illustrated by the curves for $n$ against $X$ given at the end of the paper.

From inspection of (51) it is apparent that $\bar{n}$ will have an infinite root when

$$
D+E \sin ^{2} \phi=0 .
$$

This is equivalent to the condition

$$
\epsilon_{\mathrm{I}}+\epsilon_{\mathrm{III}} \sin ^{2} \phi=0 .
$$

For the case with collisions, the condition (56) turns out to be fairly complicated, but, if we neglect collisions, it yields a simple relation analogous to that of Hines [1957].

$$
\begin{aligned}
\left\{1-\frac{\omega_{0}^{2}}{\omega^{2}}(1+\gamma)\right\} \cos ^{2} \phi+ & \left\{1+\frac{\omega_{0}^{2}}{s_{2}^{2}-\omega^{2}}\right. \\
& \left.+\frac{\gamma \omega_{0}^{2}}{s_{1}^{2}-\omega^{2}}\right\} \sin ^{2} \phi=0 .
\end{aligned}
$$

It may be noted that the condition (57) for infinite root of $n$ is dependent on $\phi$. When $\phi=\pi / 2$, an infinite root of $n$ occurs at

$$
X=\frac{\left(1-Y^{2}\right)\left(1-\gamma^{2} Y^{2}\right)}{(1+\gamma)\left(1-\gamma Y^{2}\right)} .
$$

This value is somewhat different from that obtained by Budden [1961], even if an allowance for his approximation is introduced in our computation. For the low frequency end of the whistler spectrum we consider the case $s_{2}>>\omega>>s_{1}$. In this limit the condition (56) simplifies to

$$
\left\{1-\frac{\omega_{0}^{2}}{\omega^{2}}(1+\gamma)\right\} \cos ^{2} \phi+\left\{1+\frac{\omega_{0}^{2}}{s_{2}^{2}}-\frac{\gamma \omega_{0}^{2}}{\omega^{2}}\right\} \sin ^{2} \phi=0 .
$$

If the coefficients of $\cos ^{2} \phi$ and $\sin ^{2} \phi$ in (59) are both negative, the condition for an infinity will not hold; which means that the wave can propagate in all directions. The first coefficient will be negative if $\omega<\omega_{0}$; also the second coefficient will be negative only when $\omega<\omega_{0} \sqrt{\frac{s_{1} s_{2}}{s_{2}^{2}+\omega_{0}^{2}}}$. These bounds on $\omega$ will

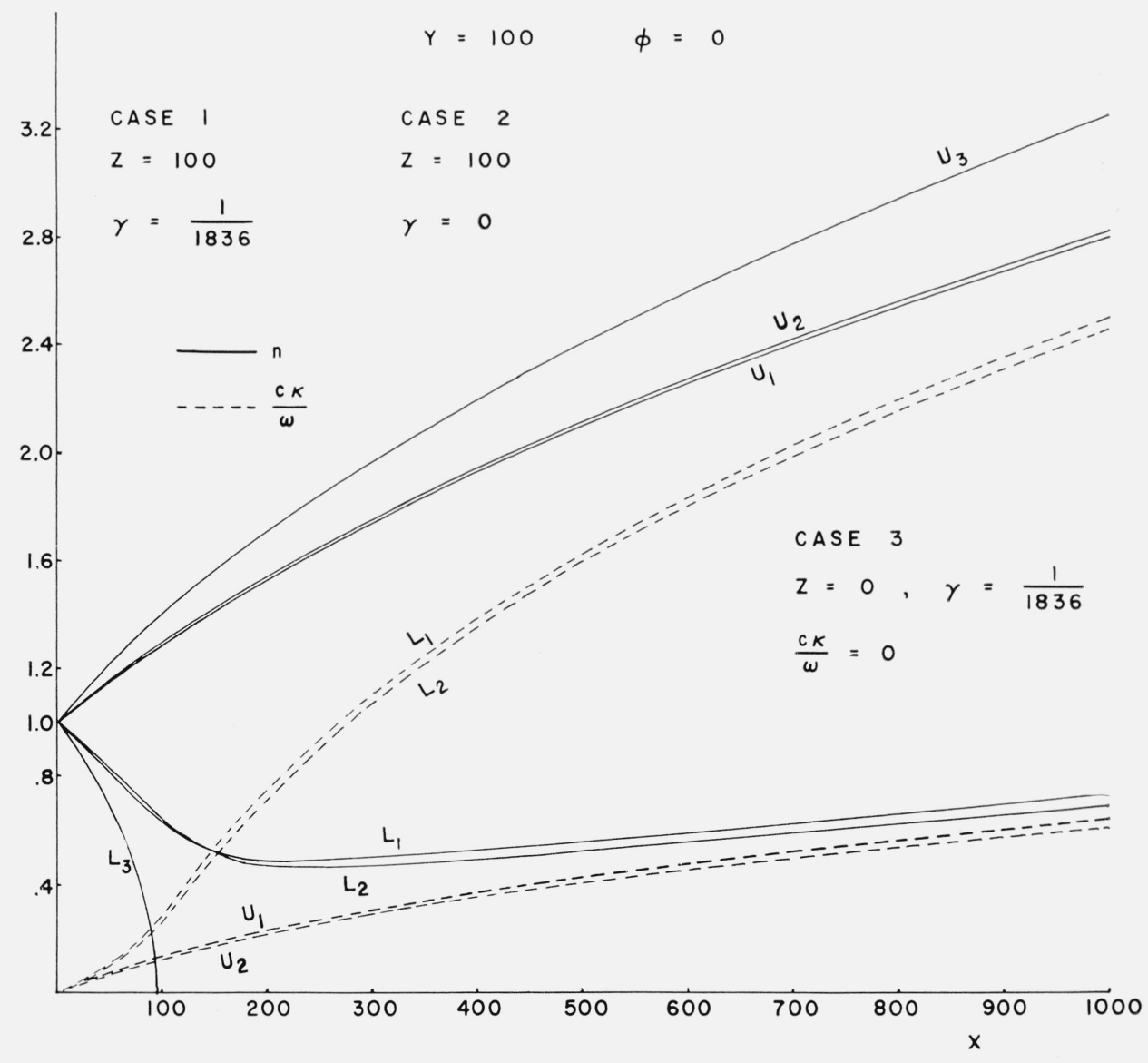

Figure 1. 
be helpful for the detection of ions with whistlers $\left(\omega<<\omega_{0}\right)$ which are propagated to a large extent in the upper atmospheric regions where our present model of a fully ionized plasma will be a close approximation [Hines, 1957].

\section{Numerical Applications}

The real refractive index, $n$, and the absorption factor, $\frac{c \kappa}{\omega}$, were evaluated from the relations

where from

$$
\frac{c^{2}}{u^{2}}=\left(n-i \frac{c \kappa}{\omega}\right)^{2}=M-i N
$$

$$
\begin{gathered}
n=\frac{1}{\sqrt{2}} \sqrt{M+\sqrt{M^{2}+N^{2}}} \\
\frac{c \kappa}{\omega}=\frac{1}{\sqrt{2}} \sqrt{-M+\sqrt{M^{2}+N^{2}}} .
\end{gathered}
$$

We note that our signs in (49) for the complex refractive index are opposite to those of the magnetoionic theory; thus our upper $(U)$ and lower $(L)$ signs, correspond respectively to the extraordinary and ordinary waves of the magneto-ionic theory. We have computed the propagation constants $n$ and $c \kappa / \omega$ as a function of $X$ for the following representative values of:

(i) $Y=100$ (whistler mode);

(ii) $Y=\sqrt{1 / \gamma}$ (lower hybrid frequency);

(iii) $Y=1 / \gamma$ (ion gyroresonance) ;

and (iv) $Y=10^{4}$ (hydromagnetic mode).

We will briefly discuss below the above cases.

Case (i) $Y=100$ (whistler mode)

Longitudinal propagation $(\phi=0)$. Figures 1 and 2. The introduction of ion motion has little effect on the birefringence, which is only slightly decreased. The important parameter for collisional effects seems to be the ratio $Z / Y$. Even for small values of this ratio ( 0.1 in fig. 2$)$, the cutoff in the ordinary ray is removed. The absorption of the extraordinary ray is increased with increasing $Z=\bar{\nu} / \omega$. The reverse is the case with the ordinary ray.

Transverse propagation $(\phi=\pi / 2)$. Figure $3 . L_{3}$ is not drawn in the figure. It follows very closely the vertical axis starting from the value $n=1$ at $X=0$, with a cutoff at $x=0.995$. The effect of ion motion is negligible for the ordinary ray. It is quite marked for the extraordinary. The birefringence of the medium is appreciably decreased. The collisions remove the cutoff for both the ordinary and extraordinary rays. The inclusion of ion motion has no effect on the absorption of the ordinary ray. For the extraordinary ray, it at first increases and then decreases the absorption. The ion motion seems to have appreciable effect for transverse

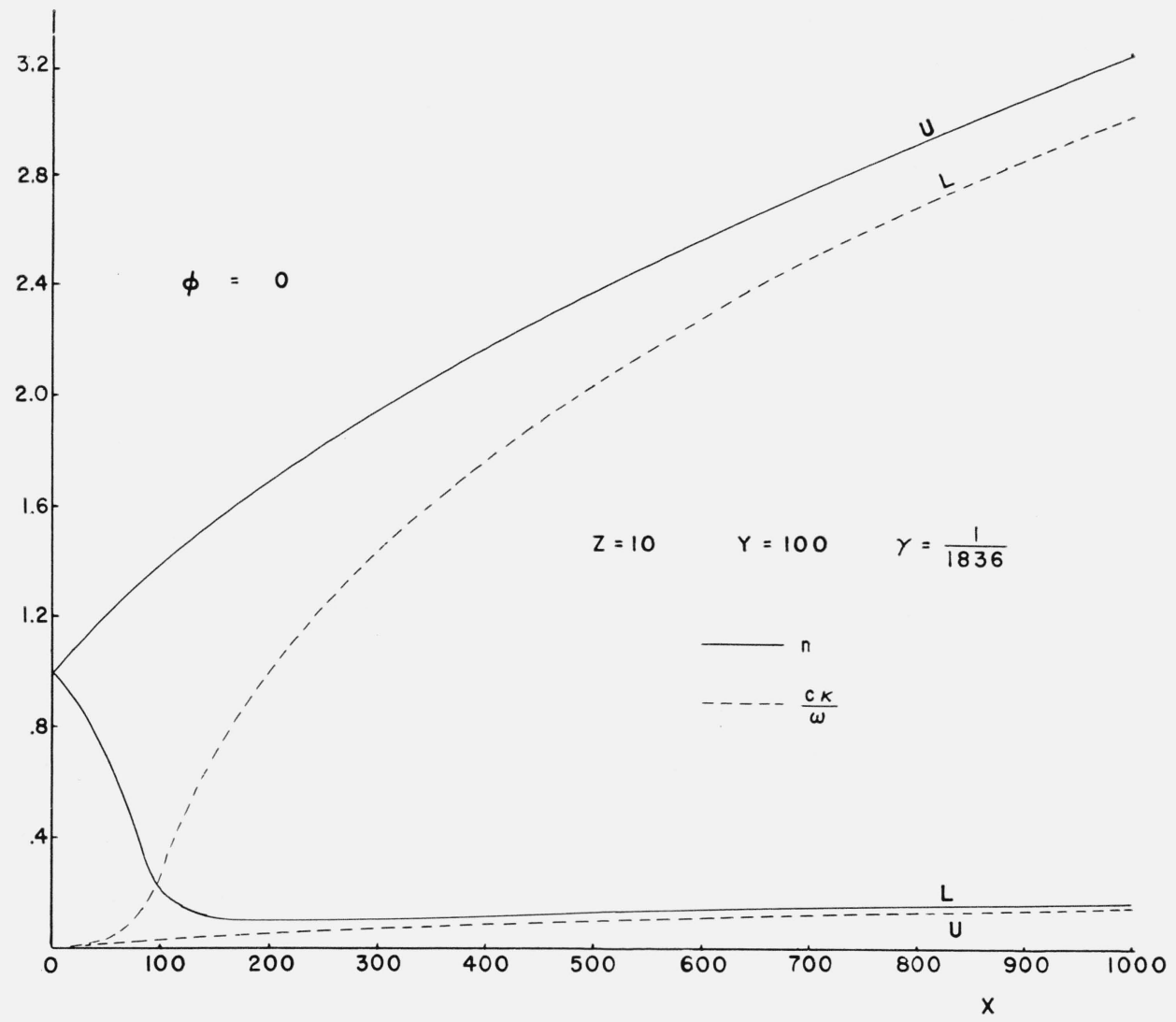

Figure 2. 


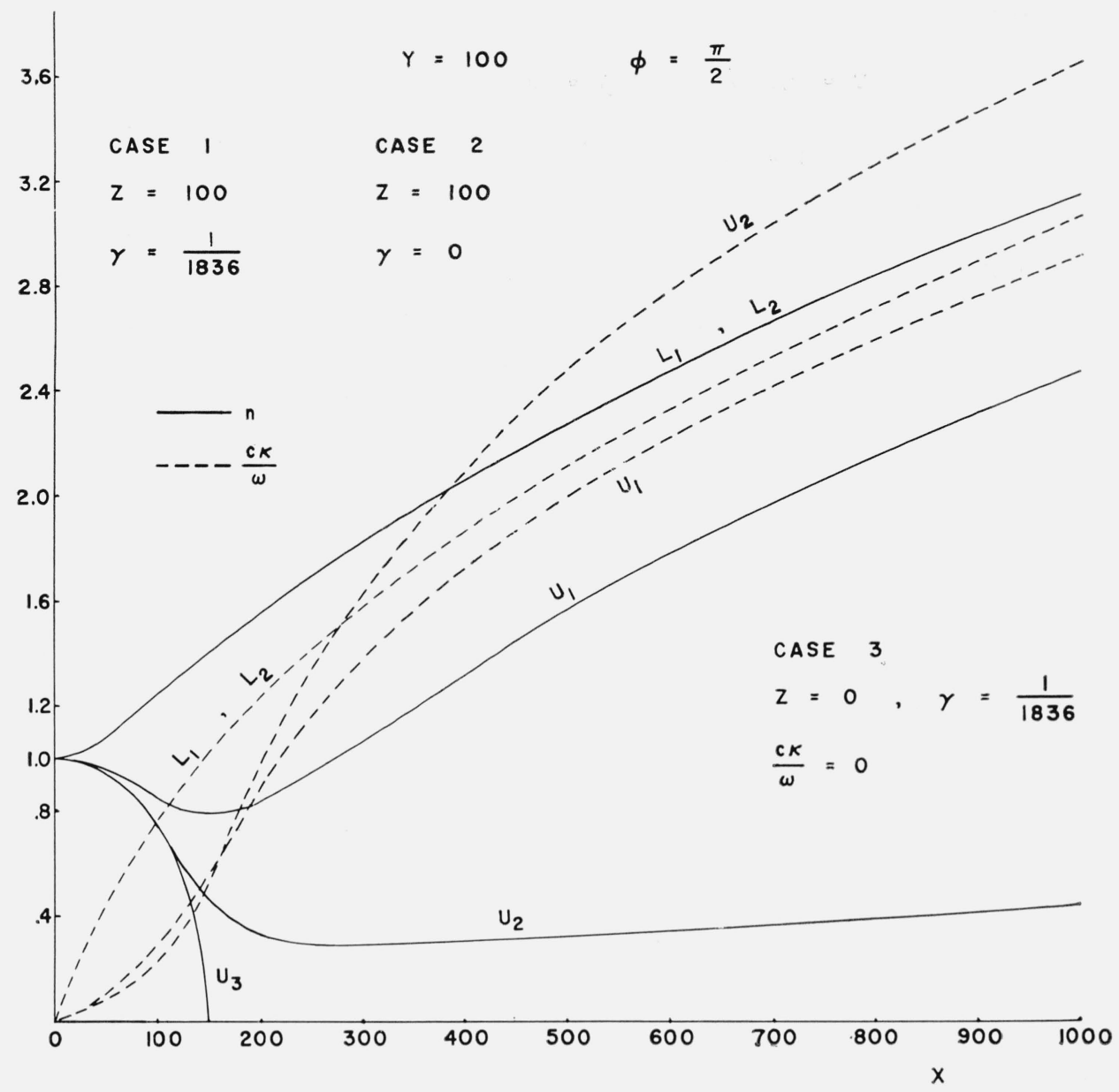

Figure 3.

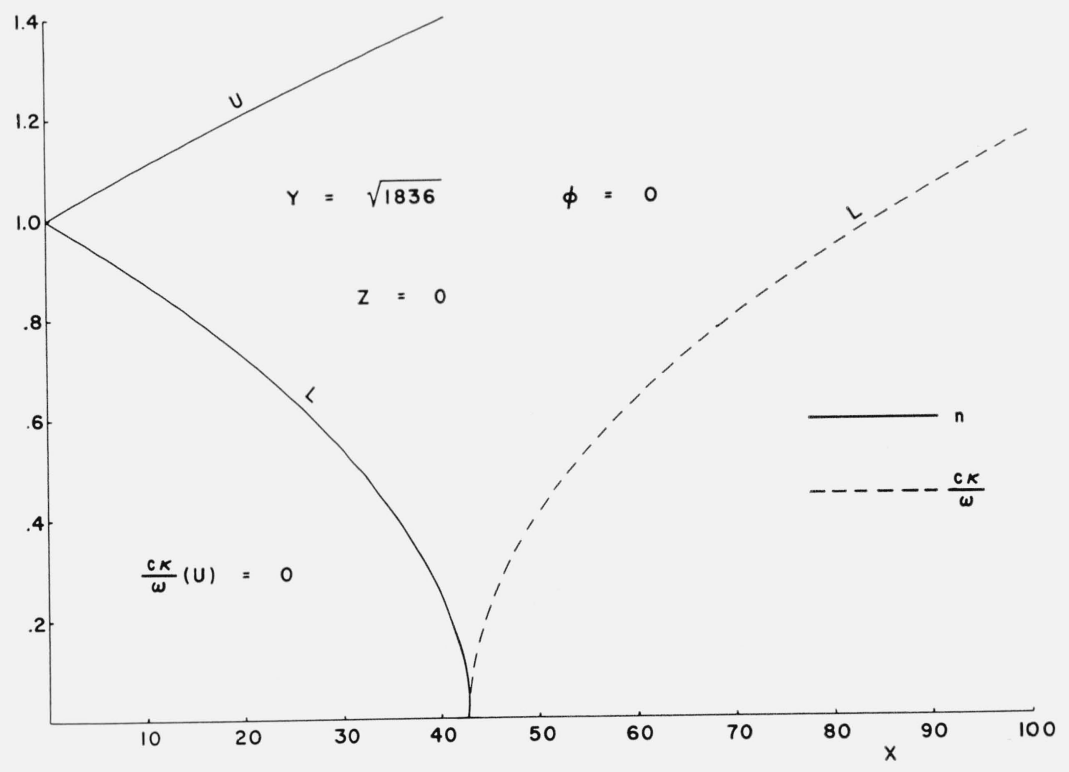

Figure 4. 


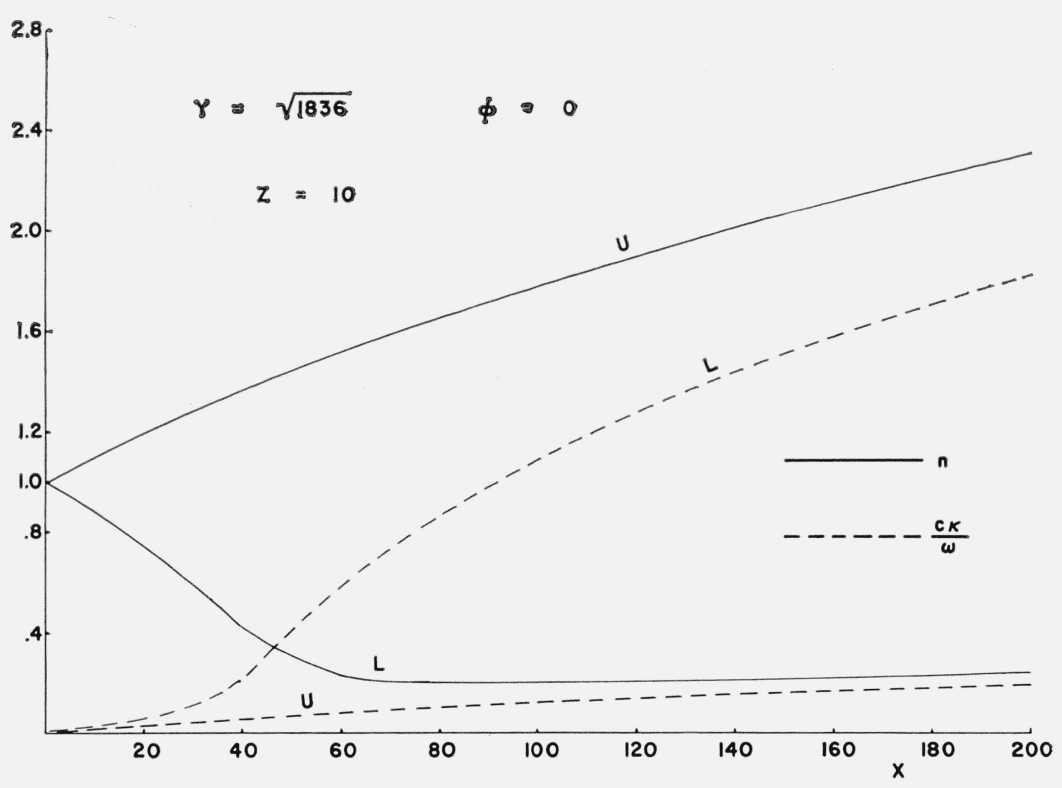

Figure 5.

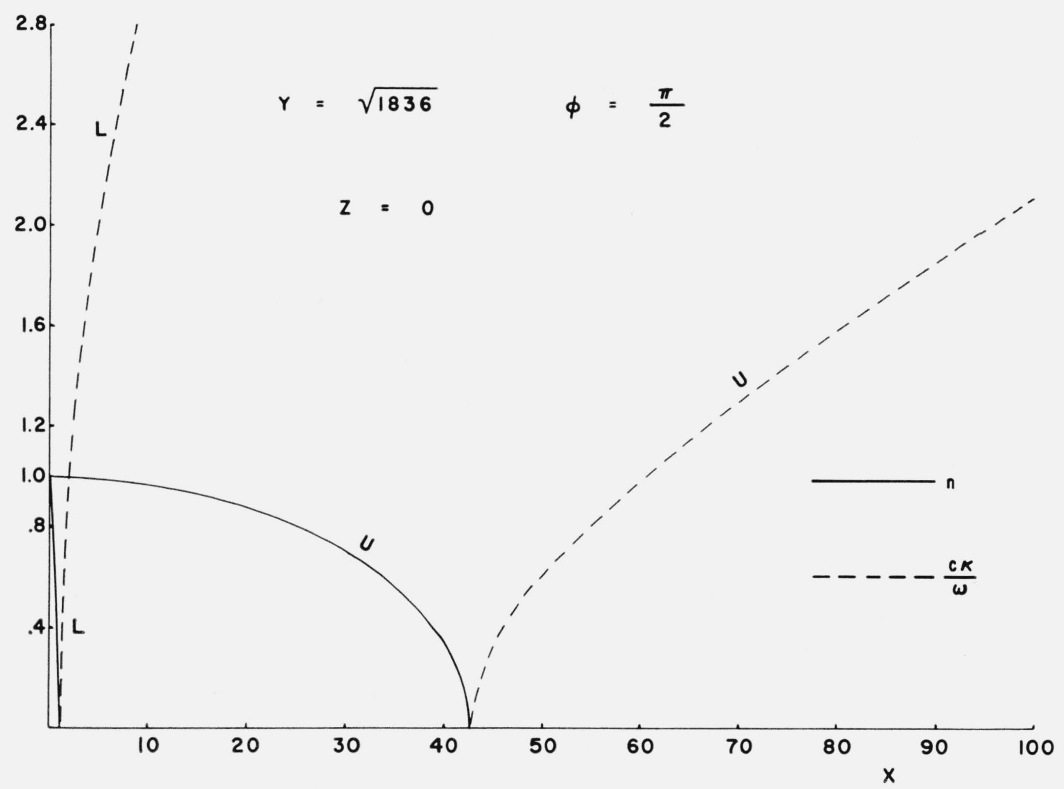

Figure 6.

propagation. The effect may well be confined within a narrow cone.

Case (ii) $Y=\sqrt{1 / \gamma}$ (lower hybrid frequency). Figures 4-7. The behavior of the propagation factors for the longitudinal case is as in the magneto-ionic theory. But for transverse propagation, collisions not only remove the cutoff but reverse the trend of the real refractive index curve (figs. 6 and 7) for the lower sign (ordinary ray of the magneto-ionic theory). How the introduction of ion motion makes possible all directions of propagation for this case has been discussed at the end of section 5 .
Case (iii) $Y=1 / \gamma$ (ion gyroresonance). Figures 8-10.

Longitudinal propagation $(\phi=0)$. We shall show next how for longitudinal propagation the ion-gyroresonance introduces a singularity in the lower mode. Figure 8 shows that even a small amount of collision removes this singularity. The wave can propagate but is nevertheless subject to high absorption. With increasing collision frequency, the absorption of the lower mode decreases, whereas that of the upper mode increases (fig. 9). 


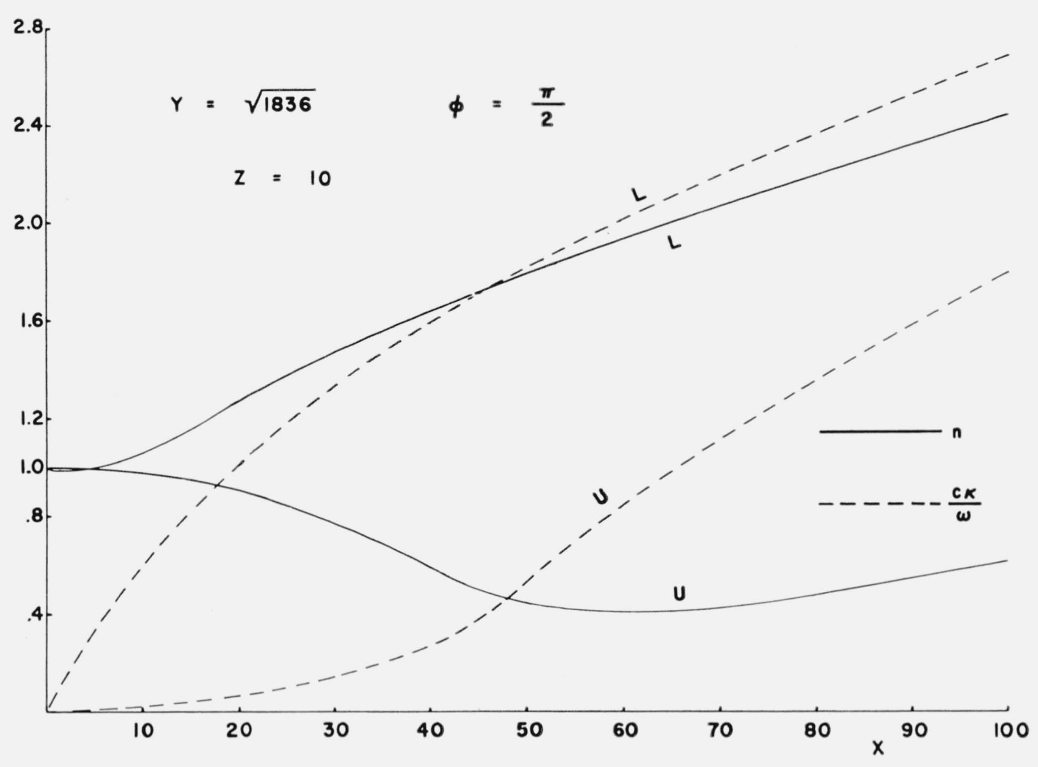

Figure 7.

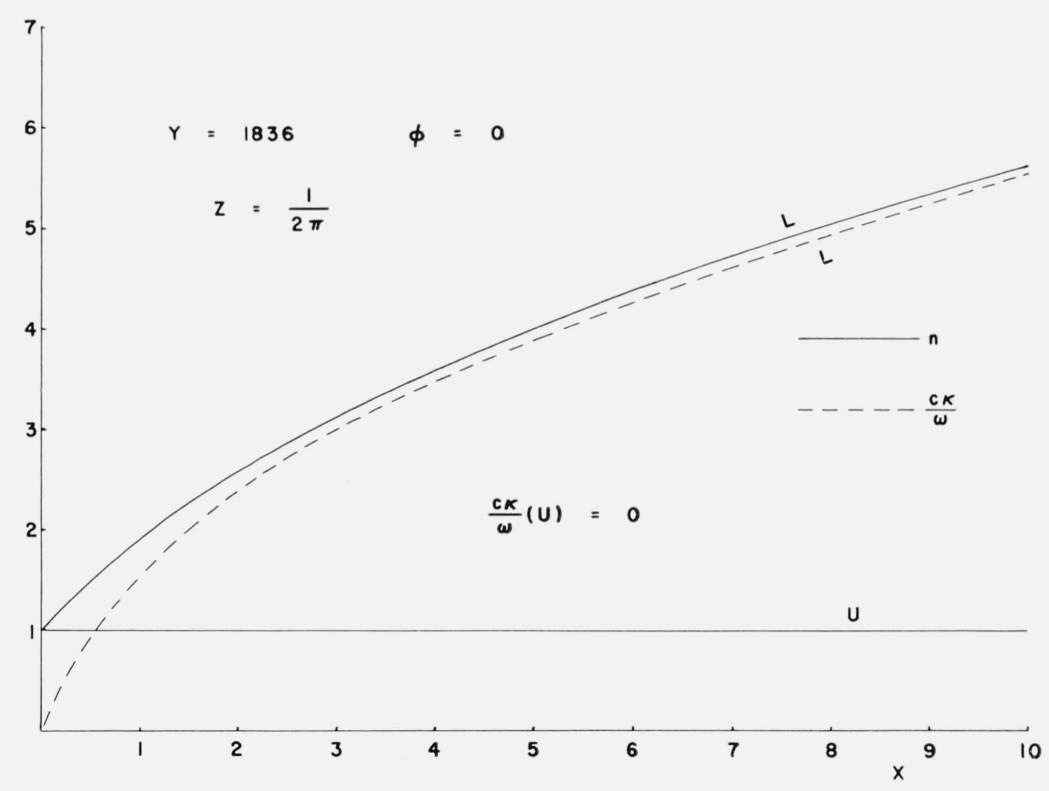

Figure 8.

Transverse propagation $(\phi=\pi / 2)$. The singularity is removed in this case and the wave can propagate. The cutoff frequencies for the two modes for no collisions is discussed below. The propagation factors for a high collision case $(Z=$ 1000) are shown in figure 10.

Case (iv) $Y=10^{4}$ (hydromagnetic mode).

Longitudinal propagation $(\phi=0)$. Figures 11 and 12 . These are the retarded magneto-acoustic mode (lower sign) and the oblique Alfvén mode (upper sign) of Denisse and Delcroiz [1961a]. Note in figure 12 that the oblique Alfvén wave does not suffer absorption, but that the retarded magnetoacoustic mode does. This property may have important application in the solar corona [Osterbrook 1961].

Transverse propagation $(\phi=\pi / 2)$. Figures 13 and 14 . The lower mode suffers a cutoff at $X \approx 1$, as in the magneto-ionic theory, which is removed by collisions. Note that the nonabsorptivity of the Alfven mode (upper sign) persists even in transverse propagation. 


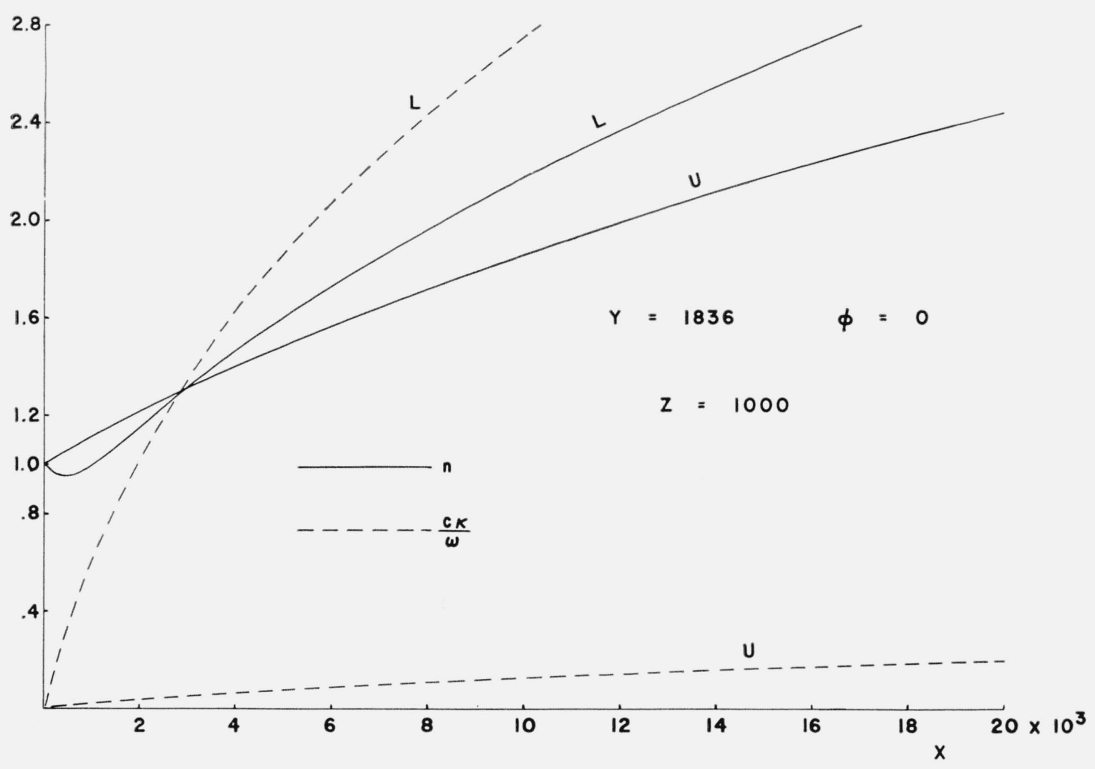

Figure 9.

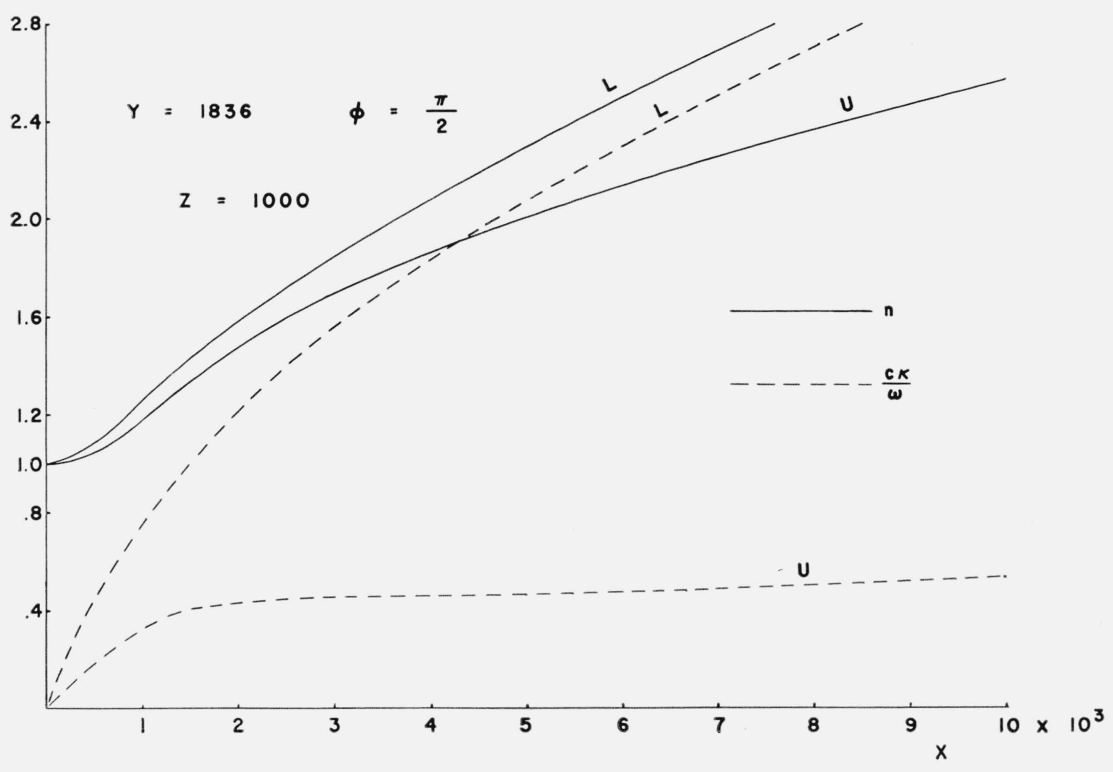

Figure 10.

We shall now give a brief analysis of the ion gyroresonance referred to in case (iii) above. From (49) we have for longitudinal propagation $(\phi=0)$ : $\bar{n}^{2}=(A+i C) / D$. Taking the minus sign and $Z=0$ (no collisions), we have $\bar{n}^{2}=n^{2}=1-X\left[(1+Y)^{-1}+\right.$ $\left.\gamma\left(1-Y_{1}\right)^{-1}\right]$, where $Y_{1}=s_{1} / \omega$. There is therefore a singularity in $n$ at the ion gyroresonance $\left(Y_{1}=1\right)$. It can be shown that a finite $Z$ will remove the singularity. For transverse propagation $(\phi=\pi / 2)$, we have $\bar{n}^{2}=(A+B \pm B) /(D+E)$. Taking the + sign, we get after a little algebra (for $Z=0$, i.e., no collisions):

$$
n^{2}=1-(1+\gamma) \frac{\left(1-Y_{1}\right) X-(1+\gamma) X^{2}}{\left(1-Y^{2}\right)\left(1-Y_{1}^{2}\right)-(1+\gamma)\left(1-Y_{1}\right) X} .
$$

When $Y_{1}=1$ (ion-gyroresonance), $n^{2} \rightarrow 2+\gamma X$. Thus $n^{2} \rightarrow 2$, as $X \rightarrow 0$. It can be shown that with collisions $n^{2} \rightarrow 1$, as $X \rightarrow 0$. (See fig. 10.) 


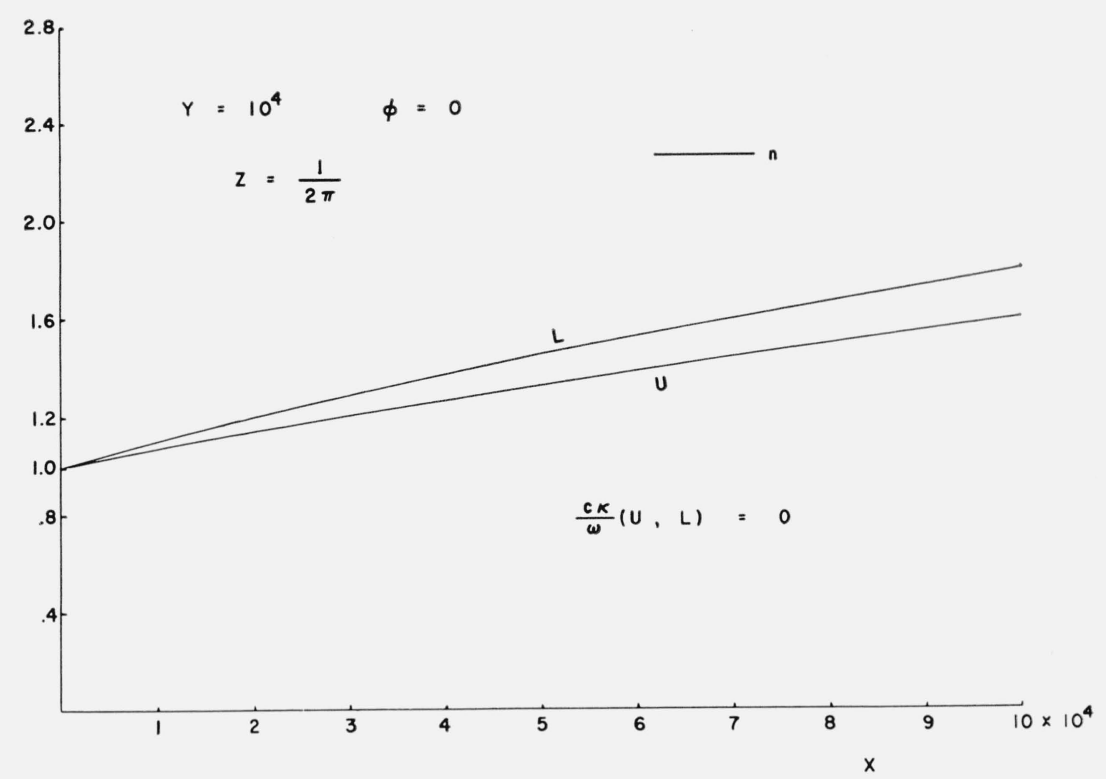

Figure 11.

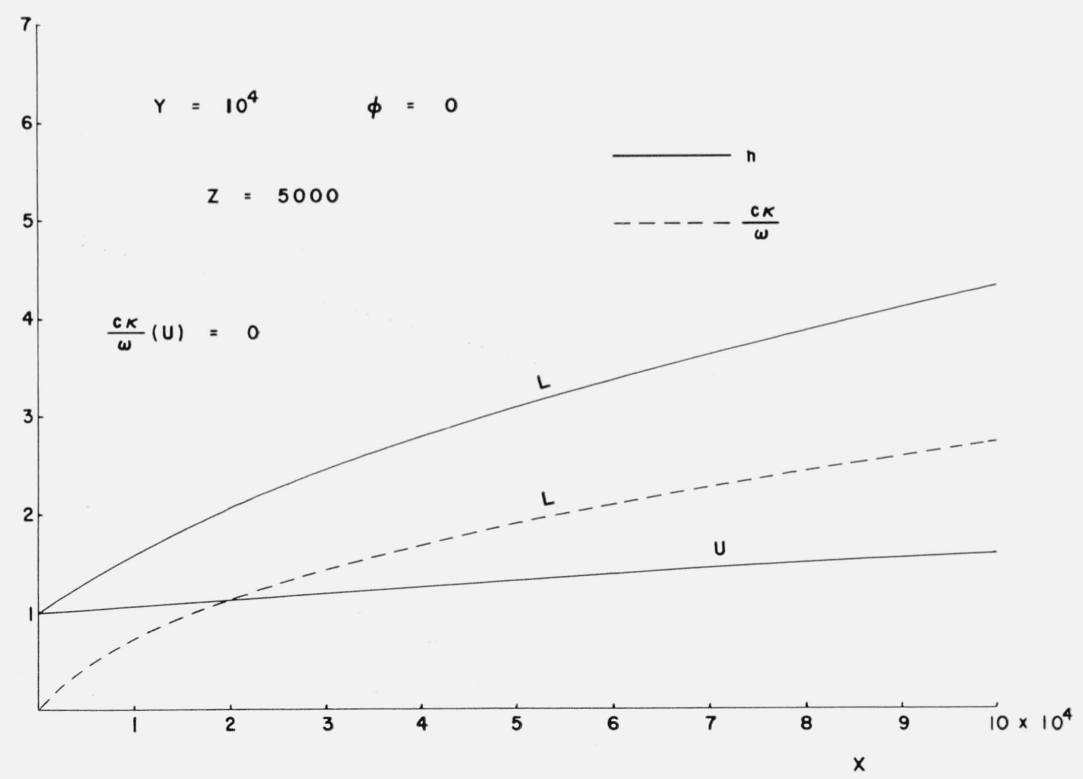

Figure 12.

\section{Conclusion}

In the present paper we have given a microscopic hydromagneto-ionic theory, to Chapman and Cowling's second approximation, of a completely ionized hydrogen plasma. As a special case for no collisions, i.e., $\bar{\nu}=0$, our (49) reduces to the expressions derived by Aström [1951] for the longitudinal and transverse components. Furthermore the most general dispersion formula derived rigorously from Denisse and Delcroix's general conditions [1961b] gives the same transverse and longitudinal modes of propagation as those obtained from our formula. As already stated in the introduction we have further agreements with the results obtained by Kantor [1963] in the limit of no collisions. All these provide an indirect check on the internal consistency of our theory. 


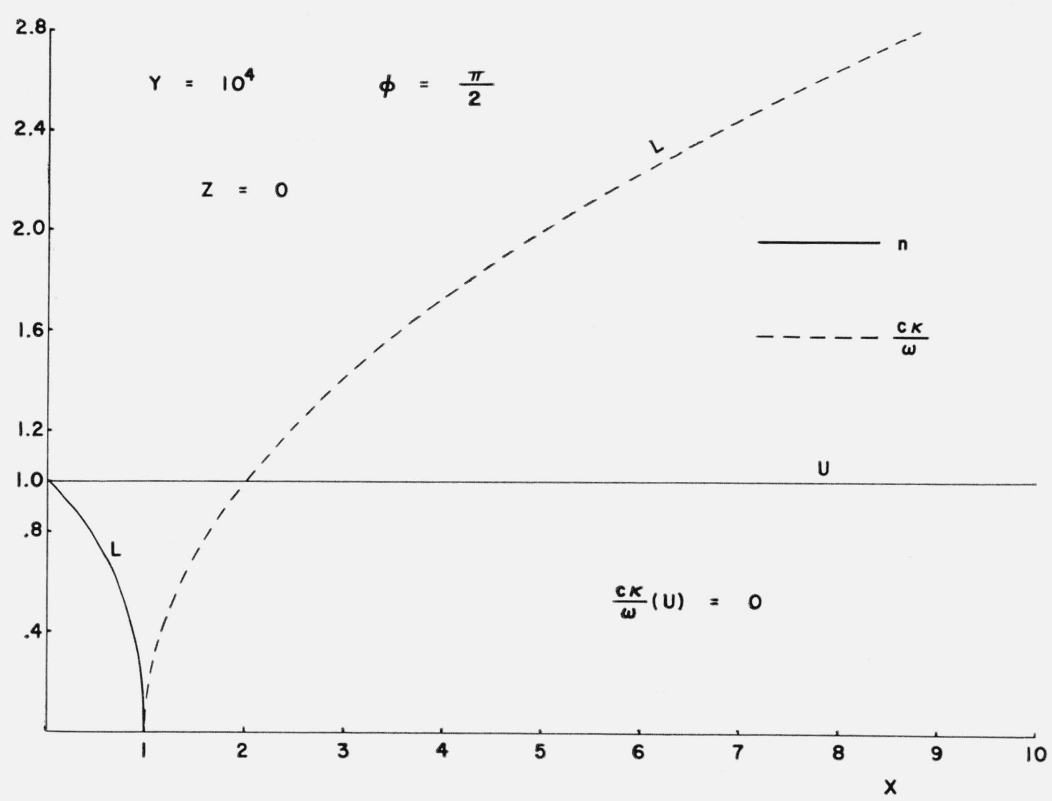

Figure 13.

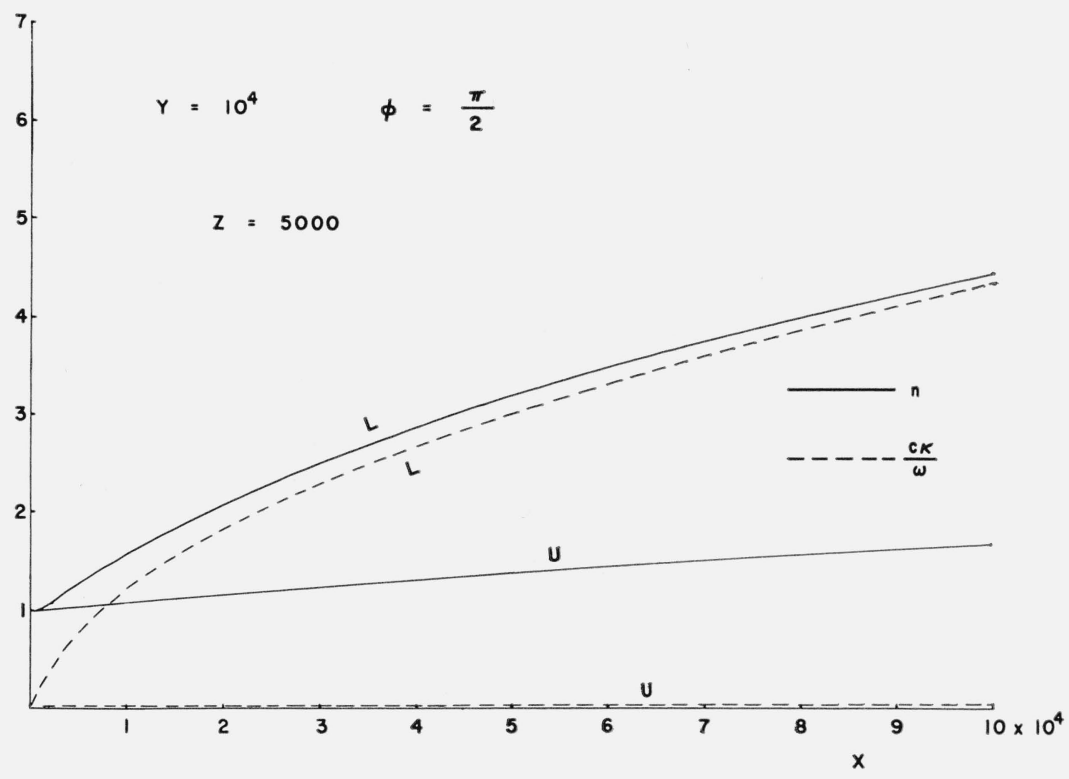

Figure 14.

We note that the treatment can easily be extended to a neutral plasma of any degree of ionization. In its present formulation, it should have important applications to hydromagnetic wave dissipation in the solar corona. The treatment of collisions in our hydromagneto-ionic theory is broad enough to include any velocity dependence of the collision cross sections. For applications to hydromagnetic wave dissipation in the terrestrial ionosphere, our treatment should be extended to a partially ionized gas. Burgers' formalism should be adequate for this extended treatment, as is evidenced by Pipkin's work [1961] on the d-c conductivity of a partially ionized gas.

The numerical applications that have been given here are merely illustrative and by no means exhaustive of the results that can be obtained from the theory.

It is a pleasure to acknowledge our indebtedness to Drs. Pfister, Poeverlein, Kantor, Prasad, and Lt. Finn for fruitful discussions, and to Mr. Arnold Shickman for the computations. 


\section{References}

Allis, W. P. (1956), Motions of Ions and Electron Handbuch der Physik 21, 383.

Allis, W. P., and J. L. Delcroix (1963), Proc. of Paris Summer School on Plasma Physics 1, 45.

Aström, E. (1951), On waves in an ionized gas, Arkiv for Fysik 2, 443.

Budden, K. G. (1961), Radio waves in the ionosphere, p. 82 (Cambridge University Press).

Burgers, J. M. (1958a), Selected topics from the theory of gas flow at high temperatures, Technical Notes BN-124 a, b, Institute for Fluid Dynamies and Applied Mathematics, University of Maryland.

Burgers, J. M. (1958b), Selected topies from the theory of gas flow at high temperatures, Technical Notes BN-124 a, b, Institute for Fluid Dynamics and Applied Mathematics, University of Maryland, Part II, pp. 65 and 93.

Burgers, J. M. (1958c), Selected topics from the theory of gas flow at high temperatures, Technical Notes BN-124 a, b, Institute for Fluid Dynamics and Applied Mathematics, University of Maryland, Part II, p. 20 equation 37, p. 42 equation 38 , and p. 58 equation 106.

Chapman, S., and T. G. Cowling (1958), The mathematical theory of non-uniform gases, $2 \mathrm{~d}$ ed., p. 56 (The University Press, Cambridge, England).

Denisse, J. F., and J. L. Delcroix (1961a), Théorie des ondes dans les plasmas, p. 85 (Dunod, Paris, France).

Denisse, J. F., and J. L. Delcroix (1961b), Théorie des ondes dans les plasmas, p. 24 equation 7 (Dunod, Paris, France).

Dungey, J. W. (1951), Derivation of the dispersion equation for Alfvén's magnetohydrodynamic waves from Bailey's electromagneto-ionic theory, Nature 16\%, 1029.

Fejer, J. A. (1960), Hydromagnetic wave propagation in the ionosphere, J. Atmospheric Terrest. Phys. 18, 135.

Gershman, B. N., V. L. Ginzburg, and N. G. Denisov (1957), Rasprostranerye elektromagnetik vele $\mathrm{v}$ plazme (ionosfere), Uspekhi Fiz. Nauk. 61, 561 (AEC-tr-3493).

Hines, C. O. (1953), Generalized magnetohydrodynamic formulae, Proc. Cambridge Phil. Soc. 49, 299.

Hines, C. O. (1957), Heavy-ion effects in audio-frequency radio propagation, J. Atmospheric Terrest. Phys. 11, 37.

Kantor, G. (1963), Whistler-hydromagnetic extension of magneto-ionic theory, AFCRL-63-929, Dec. 1963.

Lehnert, B. (1959), Plasma physics on cosmical and laboratory scale, Nuovo Cimento Suppl. 13, 59.

Leinbach, H. (1962), Interpretations of the time variations of polar cap absorption associated with solar cosmic ray bombardment, Scientific Report No. 3, Geophysical Institute, University of Alaska, UAG-R127, chapter III.

Marshall, W. (1957), The kinetic theory of an ionized gas, A.E.R.E. Reports T/R 2247, 2352, 2419, Harwell.

Menzel, D. H. (1961), Mathematical Physics, p. 325 (Dover Publications, Inc., New York, N.Y.).

Osterbrock, D. E. (1961), The heating of the solar chromosphere, plages and corona, by magnetohydrodynamic waves, Astrophys. J. 134, 347.
Piddington, J. H. (1956), Solar atmospheric heating by hydromagnetic waves, Monthly Notices Roy. Astron. Soc. 116, 314.

Pipkin, A. C. (1961), Electrical conductivity of partially ionized gases, Phys. Fluids 4, 154.

Rosenbluth, M. N., and A. N. Kaufman (1958), Plasma diffusion in a magnetic field, Phys. Rev. 109, 1 .

Schlüter, A. (1950), Dynamik des plasmas I, grundgleichungen, plasma in gekreuzten Feldern, Zeitsch.f. Naturforsch. 5a, 72 .

Schlüter, A. (1951), Dynamik des plasmas II, plasma mit neutralgas, Zeitsch. f. Naturforsch. 6a, 73.

Sen, H. K., and A. A. Wyller (1960a), On the generalization of the Appleton-Hartree magneto-ionic formulas, J. Geophys. Res. 65, No. 12, 3938.

Sen, H. K., and A. A. Wyller (1960b), On the generalization of the Appleton-Hartree magneto-ionic formulas, J. Geophys. Res. 65, No. 12, 3942-3943.

Spitzer, L. (1962), Physics of Fully Ionized Gases, 2d. ed., p. 138 (Interscience Publishers Inc., New York, N.Y.).

Wyller, A. A. (1961), Dispersion relations in a fully ionized hydrogen plasma, Proc. Fif th Internat. Conf. on Ionization Phenomena in Gases, Munich, p. 940 (North-Holland Publishing Co., Amsterdam).

Wyller, A. A. (1963a), True, ordinary and effective thermal conductivity tensors for a Lorentz-Type hydrogen gas, Astrophysica Norvegica VIII, No. 3, 53.

\section{Additional Related References}

Alfvén, H. (1947), Granulation, magnetohydrodynamic waves, and the heating of the solar corona, Monthly Notices Roy. Astron. Soc. 10\%, 211.

Akasofu, S. (1960), On the ionospheric heating by hydromagnetic waves connected with geomagnetic micropulsations, J. Atmospheric Terrest. Phys. 18, 160.

Dessler, A. J. (1959), Ionospheric heating by hydromagnetic waves, J. Geophys. Res. 64, 397.

Francis, W. E., A. J. Dessler, and R. Karplus (1960), Attenuation of hydromagnetic waves in the ionosphere, Bull. Am. Phys. Soc. 5, 316.

Hulst, H. C. van de (1953), The Sun, ed. G. P. Kuiper, Chapter 5 (University of Chicago Press, Chicago, Ill.).

Mitra, S. K. (1952), The Upper Atmosphere, p. 628 (Royal Asiatic Society of Bengal, Calcutta).

Wyller, A. A. (1963), Complete collisional effects in the thermal conductivity tensors for a fully ionized hydrogen gas, Astrophysica Norvegica VIII, No. 4, 79. 